\title{
Select Basic Science Abstracts
}

Byrom, N. C., \& Murphy, R. A. (2014). Sampling capacity underlies individual differences in human associative learning. Journal of Experimental Psychology: Animal Learning and Cognition, 40(2), 133-143. doi:10.1037/xan0000012

Though much work has studied how external factors, such as stimulus properties, influence generalization of associative strength, there has been limited exploration of the influence that internal dispositions may contribute to stimulus processing. Here we report 2 studies using a modified negative patterning discrimination to test the relationship between global processing and generalization. Global processing was associated with stronger negative patterning discrimination, indicative of limited generalization between distinct stimulus compounds and their constituent elements. In Experiment 2 , participants pretrained to adopt global processing similarly showed strong negative patterning discrimination. These results demonstrate considerable individual difference in capacity to engage in negative patterning discrimination and suggest that the tendency toward global processing may be one factor explaining this variability. The need for models of learning to account for this variability in learning is discussed.

Hahn, A., Judd, C. M., Hirsh, H. K., \& Blair, I. V. (2013, December 2). Awareness of Implicit Attitudes. Journal of Experimental Psychology: General. Advance online publication. doi:10.1037/a0035028

Research on implicit attitudes has raised questions about how well people know their own attitudes. Most research on this question has focused on the correspondence between measures of implicit attitudes and measures of explicit attitudes, with low correspondence interpreted as showing that people have little awareness of their implicit attitudes. We took a different approach and directly asked participants to predict their results on upcoming Implicit Association Test (IAT) measures of implicit attitudes toward 5 social groups. We found that participants were surprisingly accurate in their predictions. Across 4 studies, predictions were accurate regardless of whether implicit attitudes were described as true attitudes or culturally learned associations (Studies 1 and 2), regardless of whether predictions were made as specific response patterns (Study 1) or as conceptual responses (Studies 2-4), and regardless of how much experience or explanation participants received before making their predictions (Study 4). Study 3 further suggested that participants' predictions reflected unique insight into their own implicit responses, beyond intuitions about how people in general might respond. Prediction accuracy occurred despite generally low correspondence between implicit and explicit measures of attitudes, as found in prior research. Altogether, the research findings cast doubt on the belief that attitudes or evaluations measured by the IAT necessarily reflect unconscious attitudes.

Obhi, S. S., Hogeveen, J., Giacomin, M., \& Jordan, C. H. (2013, August 19). Automatic Imitation Is Reduced in Narcissists. Journal of Experimental Psychology: Human Perception and Performance. Advance online publication. doi:10.1037/a0034056

Narcissism is a personality trait that has been extensively studied in normal populations. Individuals high on subclinical narcissism tend to display an excessive self-focus and reduced concern for others. Does their disregard of others have roots in low-level processes of social perception? We investigated whether narcissism is related to the automatic imitation of observed actions. In the automatic imitation task, participants make cued actions in the presence of action videos displaying congruent or incongruent actions. The difference in response times and accuracy between congruent and incongruent trials (i.e., the interference effect) is a behavioral index of motor resonance in the brain-a process whereby observed actions activate matching motor representations in the observer. We found narcissism to be negatively related to interference in the automatic imitation task, such that high narcissism is associated with reduced imitation. Thus, levels of narcissism predict differences in the tendency to automatically resonate with others, and the pattern of data we observe suggests that a key difference is that high narcissists possess an improved ability to suppress automatic imitation when such imitation would be detrimental to task performance. To the extent that motor resonance is a product of a human mirror system, our data constitute evidence for a link between narcissistic tendencies and mirror system functioning.

Sánchez-Navarro, J. P., Driscoll, D., Anderson, S. W., Tranel, D., Bechara, A., \& Buchanan, T. W. (2014). Alterations of attention and emotional processing following childhood-onset damage to the prefrontal cortex. Behavioral Neuroscience, 128(1), 1-11. doi:10.1037/a0035415

The prefrontal cortex (PFC), especially the medial sector, plays a crucial role in emotional processing. Damage to this region results in impaired processing of emotional information, perhaps attributable to an inability to initiate and maintain attention toward emotional materials, a process that is normally automatic. Childhood onset damage to the PFC impairs emotional processing more than adult-onset PFC damage. The aim of this work was to study the involvement of the PFC in attention to emotional stimuli, and to explore how age at lesion onset affects this involvement. To address these issues, we studied both the emotional and attentional modulation of the startle reflex. Our sample was composed of 4 patients with childhood-onset PFC damage, 6 patients with adult-onset PFC damage, and 10 healthy comparison participants. Subjects viewed 54 affective pictures; acoustic startle probes were presented at $300 \mathrm{~ms}$ after picture onset in 18 pictures (as an index of attentional modulation) and at 3,800 $\mathrm{ms}$ after picture onset in 18 pictures (as an index of emotional modulation). Childhood-onset PFC patients did not show attentional or emotional modulation of the response, in contrast to adult-onset PFC damage and comparison participants. Early onset damage to the PFC results, therefore, in more severe dysfunction in the processing of affective stimuli than adult-onset PFC damage, perhaps reflecting limited plasticity in the neural systems that support these processes.

Guenther, A., Brust, V., Dersen, M., \& Trillmich, F. (2014). Learning and personality types are related in cavies (Cavia aperea). Journal of Comparative Psychology, 128(1), 74-81. doi:10.1037/a0033678

The evolution and maintenance of consistent individual differences, so called animal personalities, have attracted much research interest over the past decades. Variation along common personality traits, such as boldness or exploration, is often associated with risk-reward trade-offs. Individuals that are bolder and hence take more risks may be more successful in acquiring resources over the short term. Cautious individuals taking fewer risks may, on the other hand, live longer, but may also gather fewer resources over the short term. According to recent theory, individual differences in personality may be functionally related to individual differences in cognitive performance (i.e., the way in which individuals acquire or use information). Individual differences in the acquisition speed of cognitively challenging tasks are often associated with a speed-accuracy trade-off. Accuracy can be improved by investing more time in the decision-making process or, conversely, decisions can be made more quickly at the cost of making more mistakes. Hence, the speed-accuracy trade-off often involves a risk-reward trade-off. We tested whether 3 personality traits, boldness, activity, and aggressiveness, are correlated with individual learning, associative learning speed, and behavioral flexibility as assessed by reversal learning in wild cavies (Cavia aperea). We found strong positive relationships between all personality traits and learning speed, whereas flexibility was negatively associated with aggressiveness. Our results support the hypothesis that performance reflects individual differences in personality in a predictable way. 